\title{
K-THEORY FOR CUNTZ-KRIEGER ALGEBRAS ARISING FROM REAL QUADRATIC MAPS
}

\author{
NUNO MARTINS, RICARDO SEVERINO, and J. SOUSA RAMOS
}

\author{
Received 20 September 2002
}

\begin{abstract}
We compute the $K$-groups for the Cuntz-Krieger algebras ${ }_{\mathcal{O}_{\mathscr{K}}\left(f_{\mu}\right)}$, where $A_{\mathscr{K}_{\ell}\left(f_{\mu}\right)}$ is the Markov transition matrix arising from the kneading sequence $\mathscr{K}\left(f_{\mu}\right)$ of the one-parameter family of real quadratic maps $f_{\mu}$.
\end{abstract}

2000 Mathematics Subject Classification: 37A55, 37B10, 37E05, 46L80.

Consider the one-parameter family of real quadratic maps $f_{\mu}:[0,1] \rightarrow[0,1]$ defined by $f_{\mu}(x)=\mu x(1-x)$, with $\mu \in[0,4]$. Using Milnor-Thurston kneading theory [14], Guckenheimer [5] has classified, up to topological conjugacy, a certain class of maps, which includes the quadratic family. The idea of kneading theory is to encode information about the orbits of a map in terms of infinite sequences of symbols and to exploit the natural order of the interval to establish topological properties of the map. In what follows, $I$ denotes the unit interval $[0,1]$ and $c$ the unique turning point of $f_{\mu}$. For $x \in I$, let

$$
\varepsilon_{n}(x)= \begin{cases}-1, & \text { if } f_{\mu}^{n}(x)>c, \\ 0, & \text { if } f_{\mu}^{n}(x)=c, \\ +1, & \text { if } f_{\mu}^{n}(x)<c .\end{cases}
$$

The sequence $\varepsilon(x)=\left(\varepsilon_{n}(x)\right)_{n=0}^{\infty}$ is called the itinerary of $x$. The itinerary of $f_{\mu}(c)$ is called the kneading sequence of $f_{\mu}$ and will be denoted by $\mathscr{K}\left(f_{\mu}\right)$. Observe that $\varepsilon_{n}\left(f_{\mu}(x)\right)=\varepsilon_{n+1}(x)$, that is, $\varepsilon\left(f_{\mu}(x)\right)=\sigma \varepsilon(x)$, where $\sigma$ is the shift map. Let $\sum=\{-1,0,+1\}$ be the alphabet set. The sequences on $\sum^{\mathbb{N}}$ are ordered lexicographically. However, this ordering is not reflected by the mapping $x \rightarrow \varepsilon(x)$ because the map $f_{\mu}$ reverses orientation on $[c, 1]$. To take this into account, for a sequence $\varepsilon=\left(\varepsilon_{n}\right)_{n=0}^{\infty}$ of the symbols $-1,0$, and +1 , another sequence $\theta=\left(\theta_{n}\right)_{n=0}^{\infty}$ is defined by $\theta_{n}=\prod_{i=0}^{n} \varepsilon_{i}$. If $\varepsilon=\varepsilon(x)$ is the itinerary of a point $x \in I$, then $\theta=\theta(x)$ is called the invariant coordinate of $x$. The fundamental observation of Milnor and Thurston [14] is the monotonicity of the invariant coordinates:

$$
x<y \Longrightarrow \theta(x) \leq \theta(y)
$$


We now consider only those kneading sequences that are periodic, that is,

$$
\begin{aligned}
\mathscr{K}\left(f_{\mu}\right) & =\varepsilon_{0}\left(f_{\mu}(c)\right) \cdots \varepsilon_{n-1}\left(f_{\mu}(c)\right) \varepsilon_{0}\left(f_{\mu}(c)\right) \cdots \varepsilon_{n-1}\left(f_{\mu}(c)\right) \cdots \\
& =\left(\varepsilon_{0}\left(f_{\mu}(c)\right) \cdots \varepsilon_{n-1}\left(f_{\mu}(c)\right)\right)^{\infty} \equiv\left(\varepsilon_{1}(c) \cdots \varepsilon_{n}(c)\right)^{\infty}
\end{aligned}
$$

for some $n \in \mathbb{N}$. The sequences $\sigma^{i}\left(\mathscr{K}\left(f_{\mu}\right)\right)=\varepsilon_{i+1}(c) \varepsilon_{i+2}(c) \cdots, i=0,1,2, \ldots$, will then determine a Markov partition of $I$ into $n-1$ line intervals $\left\{I_{1}, I_{2}, \ldots\right.$, $\left.I_{n-1}\right\}$ [15], whose definitions will be given in the proof of Theorem 1 . Thus, we will have a Markov transition matrix $A_{\mathscr{K}}\left(f_{\mu}\right)$ defined by

$$
A_{\mathscr{K}\left(f_{\mu}\right)}:=\left(a_{i j}\right) \quad \text { with } a_{i j}= \begin{cases}1, & \text { if } f_{\mu}\left(\operatorname{int} I_{i}\right) \supseteq \operatorname{int} I_{j} \\ 0, & \text { otherwise. }\end{cases}
$$

It is easy to see that the matrix $A_{\mathscr{K}\left(f_{\mu}\right)}$ is not a permutation matrix and no row or column of $A_{\mathscr{K}\left(f_{\mu}\right)}$ is zero. Thus, for each one of these matrices and following the work of Cuntz and Krieger [3], one can construct the Cuntz-Krieger algebra $\mathrm{O}_{A_{\mathscr{K}\left(f_{\mu}\right)}}$. In [2], Cuntz proved that

$$
K_{0}\left(\mathscr{O}_{A}\right) \cong \mathbb{Z}^{r} /\left(1-A^{T}\right) \mathbb{Z}^{r}, \quad K_{1}\left(\mathscr{O}_{A}\right) \cong \operatorname{ker}\left(I-A^{t}: \mathbb{Z}^{r} \longrightarrow \mathbb{Z}^{r}\right),
$$

for an $r \times r$ matrix $A$ that satisfies a certain condition (I) (see [3]), which is readily verified by the matrices $A_{\mathscr{\kappa}\left(f_{\mu}\right)}$. In [1], Bowen and Franks introduced the group $B F(A):=\mathbb{Z}^{r} /(1-A) \mathbb{Z}^{r}$ as an invariant for flow equivalence of topological Markov subshifts determined by $A$.

We can now state and prove the following theorem.

THEOREM 1. Let $\mathscr{K}\left(f_{\mu}\right)=\left(\varepsilon_{1}(c) \varepsilon_{2}(c) \cdots \varepsilon_{n}(c)\right)^{\infty}$ for some $n \in \mathbb{N} \backslash\{1\}$. Thus,

$$
\begin{gathered}
K_{0}\left(\mathcal{O}_{A_{\mathscr{K}}\left(f_{\mu}\right)}\right) \cong \mathbb{Z}_{a} \quad \text { with } a=\left|1+\sum_{l=1}^{n-1} \prod_{i=1}^{l} \varepsilon_{i}(c)\right|, \\
K_{1}\left(\mathcal{O}_{A_{\mathscr{K}}\left(f_{\mu}\right)}\right) \cong \begin{cases}\{0\}, & \text { if } a \neq 0, \\
\mathbb{Z}, & \text { if } a=0 .\end{cases}
\end{gathered}
$$

Proof. Set $z_{i}=\varepsilon_{i}(c) \varepsilon_{i+1}(c) \cdots$ for $i=1,2, \ldots$ Let $z_{i}^{\prime}=f_{\mu}^{i}(c)$ be the point on the unit interval $[0,1]$ represented by the sequence $z_{i}$ for $i=1,2, \ldots$. We have $\sigma\left(z_{i}\right)=z_{i+1}$ for $i=1, \ldots, n-1$ and $\sigma\left(z_{n}\right)=z_{1}$. Denote by $\omega$ the $n \times n$ matrix representing the shift map $\sigma$. Let $C_{0}$ be the vector space spanned by the formal basis $\left\{z_{1}^{\prime}, \ldots, z_{n}^{\prime}\right\}$. Now, let $\rho$ be the permutation of the set $\{1, \ldots, n\}$, which allows us to order the points $z_{1}^{\prime}, \ldots, z_{n}^{\prime}$ on the unit interval $[0,1]$, that is,

$$
0<z_{\rho(1)}^{\prime}<z_{\rho(2)}^{\prime}<\cdots<z_{\rho(n)}^{\prime}<1 .
$$


Set $x_{i}:=z_{\rho(i)}^{\prime}$ with $i=1, \ldots, n$ and let $\pi$ denote the permutation matrix which takes the formal basis $\left\{z_{1}^{\prime}, \ldots, z_{n}^{\prime}\right\}$ to the formal basis $\left\{x_{1}, \ldots, x_{n}\right\}$. We will denote by $C_{1}$ the $(n-1)$-dimensional vector space spanned by the formal basis $\left\{x_{i+1}-x_{i}: i=1, \ldots, n-1\right\}$. Set

$$
I_{i}:=\left[x_{i}, x_{i+1}\right] \text { for } i=1, \ldots, n-1 .
$$

Thus, we can define the Markov transition matrix $A_{\mathscr{K}\left(f_{\mu}\right)}$ as above. Let $\varphi$ denote the incidence matrix that takes the formal basis $\left\{x_{1}, \ldots, x_{n}\right\}$ of $C_{0}$ to the formal basis $\left\{x_{2}-x_{1}, \ldots, x_{n}-x_{n-1}\right\}$ of $C_{1}$. Put $\eta:=\varphi \pi$. As in [7,8], we obtain an endomorphism $\alpha$ of $C_{1}$, that makes the following diagram commutative:

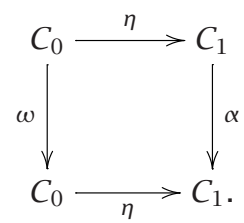

We have $\alpha=\eta \omega \eta^{T}\left(\eta \eta^{T}\right)^{-1}$. Remark that if we neglect the negative signs on the matrix $\alpha$, then we will obtain precisely the Markov transition matrix $A_{\mathscr{K}\left(f_{\mu}\right)}$. In fact, consider the $(n-1) \times(n-1)$ matrix

$$
\beta:=\left[\begin{array}{cc}
1_{n_{L}} & 0 \\
0 & -1_{n_{R}}
\end{array}\right],
$$

where $1_{n_{L}}$ and $1_{n_{R}}$ are the identity matrices of ranks $n_{L}$ and $n_{R}$, respectively, with $n_{L}\left(n_{R}\right)$ being the number of intervals $I_{i}$ of the Markov partition placed on the left- (right-) hand side of the turning point of $f_{\mu}$. Therefore, we have

$$
A_{\mathscr{K}\left(f_{\mu}\right)}=\beta \alpha
$$

Now, consider the following matrix:

$$
\gamma_{\mathscr{K}\left(f_{\mu}\right)}:=\left(\gamma_{i j}\right) \text { with } \begin{cases}\gamma_{i i}=\varepsilon_{i}(c), & i=1, \ldots, n, \\ \gamma_{i n}=-\varepsilon_{i}(c), & i=1, \ldots, n, \\ \gamma_{i j}=0, & \text { otherwise. }\end{cases}
$$

The matrix $\gamma_{\mathscr{L}}\left(f_{\mu}\right)$ makes the diagram

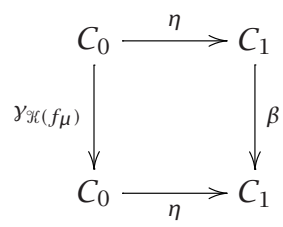


commutative. Finally, set $\theta_{\mathscr{K}\left(f_{\mu}\right)}:=\theta_{\mathscr{K}\left(f_{\mu}\right)} \omega$. Then, the diagram

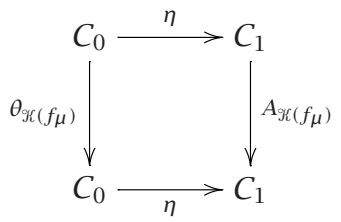

is also commutative. Now, notice that the transpose of $\eta$ has the following factorization:

$$
\eta^{T}=Y i X
$$

where $Y$ is an invertible (over $\mathbb{Z}$ ) $n \times n$ integer matrix given by

$$
Y:=\left(\begin{array}{cccccc}
1 & 0 & \ldots & & & 0 \\
0 & 1 & 0 & \ldots & & 0 \\
\vdots & 0 & \ddots & \ddots & & \vdots \\
& \vdots & & & 0 & \\
0 & 0 & \ldots & 0 & 1 & 0 \\
-1 & -1 & \ldots & & -1 & 1
\end{array}\right),
$$

$i$ is the inclusion $C_{1} \hookrightarrow C_{0}$ given by

$$
i:=\left(\begin{array}{cccc}
1 & 0 & & 0 \\
0 & \ddots & \ddots & \vdots \\
\vdots & \ddots & & 0 \\
& & & 1 \\
0 & \cdots & & 0
\end{array}\right),
$$

and $X$ is an invertible (over $\mathbb{Z})(n-1) \times(n-1)$ integer matrix obtained from the $(n-1) \times n$ matrix $\eta^{T}$ by removing the $n$th row of $\eta^{T}$. Thus, from the commutative diagram

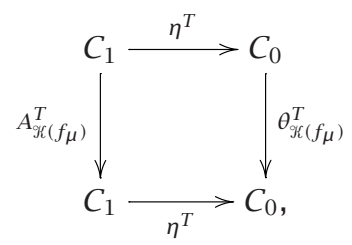


we will have the following commutative diagram with short exact rows:

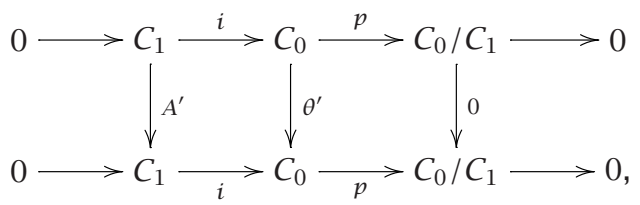

where the map $p$ is represented by the $1 \times n$ matrix $\left[\begin{array}{llll}0 & \cdots & 0 & 1\end{array}\right]$ and

$$
A^{\prime}=X A_{\mathscr{K}\left(f_{\mu}\right)}^{T} X^{-1}, \quad \theta^{\prime}=Y^{-1} \theta_{\mathscr{K}\left(f_{\mu}\right)}^{T} Y
$$

that is, $A^{\prime}$ is similar to $A_{\mathscr{K}\left(f_{\mu}\right)}^{T}$ over $\mathbb{Z}$ and $\theta^{\prime}$ is similar to $\theta_{\mathscr{K}\left(f_{\mu}\right)}^{T}$ over $\mathbb{Z}$. Hence, for example, by [10] we obtain, respectively,

$$
\begin{aligned}
\mathbb{Z}^{n-1} /\left(1-A^{\prime}\right) \mathbb{Z}^{n-1} & \cong \mathbb{Z}^{n-1} /\left(1-A_{\mathscr{K}}\left(f_{\mu}\right)\right) \mathbb{Z}^{n-1}, \\
\mathbb{Z}^{n} /\left(1-\theta^{\prime}\right) \mathbb{Z}^{n} & \cong \mathbb{Z}^{n} /\left(1-\theta_{\mathscr{K}\left(f_{\mu}\right)}\right) \mathbb{Z}^{n} .
\end{aligned}
$$

Now, from the last diagram we have, for example, by [9],

$$
\theta^{\prime}=\left[\begin{array}{rr}
A^{\prime} & * \\
0 & 0
\end{array}\right]
$$

Therefore,

$$
\begin{aligned}
\mathbb{Z}^{n-1} /\left(1-A^{\prime}\right) \mathbb{Z}^{n-1} & \cong \mathbb{Z}^{n} /\left(1-\theta^{\prime}\right) \mathbb{Z}^{n}, \\
\mathbb{Z}^{n-1} /\left(1-A_{\mathscr{K}}\left(f_{\mu}\right)\right) & \mathbb{Z}^{n-1} \cong \mathbb{Z}^{n} /\left(1-\theta_{\mathscr{K}}\left(f_{\mu}\right)\right) \mathbb{Z}^{n} .
\end{aligned}
$$

Next, we will compute $\mathbb{Z}^{n} /\left(1-\theta_{\mathscr{K}\left(f_{\mu}\right)}\right) \mathbb{Z}^{n}$. From the previous discussions and notations, the $n \times n$ matrix $\theta_{\mathscr{K}\left(f_{\mu}\right)}$ is explicitly given by

$$
\theta_{\mathscr{K}\left(f_{\mu}\right)}:=\left(\begin{array}{ccccc}
-\varepsilon_{1}(c) & \varepsilon_{1}(c) & 0 & \cdots & 0 \\
\vdots & 0 & \ddots & \ddots & \vdots \\
& \vdots & \ddots & & 0 \\
-\varepsilon_{n-1}(c) & & & & \varepsilon_{n-1}(c) \\
0 & 0 & \cdots & & 0
\end{array}\right) .
$$

Notice that the matrix $\theta_{\mathscr{K}\left(f_{\mu}\right)}$ completely describes the dynamics of $f_{\mu}$. Finally, using row and column elementary operations over $\mathbb{Z}$, we can find invertible 
(over $\mathbb{Z}$ ) matrices $U_{1}$ and $U_{2}$ with integer entries such that

$$
1-\theta_{\mathscr{K}\left(f_{\mu}\right)}=U_{1}\left(\begin{array}{cccc}
1+\sum_{l=1}^{n-1} \prod_{i=1}^{l} \varepsilon_{i}(c) & & & \\
& 1 & & \\
& & \ddots & \\
& & & 1
\end{array}\right) U_{2} .
$$

Thus, we obtain

$$
K_{0}\left(\mathcal{O}_{A_{\mathscr{K}}\left(f_{\mu}\right)}\right) \cong \mathbb{Z}^{n-1} /\left(1-A_{\mathscr{K}\left(f_{\mu}\right)}^{T}\right) \mathbb{Z}^{n-1} \cong \mathbb{Z}_{a},
$$

where

$$
a=\left|1+\sum_{l=1}^{n-1} \prod_{i=1}^{l} \varepsilon_{i}(c)\right|, \quad n \in \mathbb{N} \backslash\{1\} .
$$

EXAMPLE 2. Set

$$
\mathscr{K}\left(f_{\mu}\right)=(R L L R R C)^{\infty},
$$

where $R=-1, L=+1$, and $C=0$. Thus, we can construct the $5 \times 5$ Markov transition matrix $A_{\mathscr{K}}\left(f_{\mu}\right)$ and the matrices $\theta_{\mathscr{K}\left(f_{\mu}\right)}, \omega, \varphi$, and $\pi$ :

$$
\begin{aligned}
& A_{\mathscr{K}\left(f_{\mu}\right)}=\left(\begin{array}{ccccc}
0 & 1 & 1 & 0 & 0 \\
0 & 0 & 0 & 1 & 1 \\
0 & 0 & 0 & 0 & 1 \\
0 & 0 & 1 & 1 & 0 \\
1 & 1 & 0 & 0 & 0
\end{array}\right), \quad \theta_{\mathscr{K}\left(f_{\mu}\right)}=\left(\begin{array}{rrrrrr}
1 & -1 & 0 & 0 & 0 & 0 \\
-1 & 0 & 1 & 0 & 0 & 0 \\
-1 & 0 & 0 & 1 & 0 & 0 \\
1 & 0 & 0 & 0 & -1 & 0 \\
1 & 0 & 0 & 0 & 0 & -1 \\
0 & 0 & 0 & 0 & 0 & 0
\end{array}\right), \\
& \omega=\left(\begin{array}{llllll}
0 & 1 & 0 & 0 & 0 & 0 \\
0 & 0 & 1 & 0 & 0 & 0 \\
0 & 0 & 0 & 1 & 0 & 0 \\
0 & 0 & 0 & 0 & 1 & 0 \\
0 & 0 & 0 & 0 & 0 & 1 \\
1 & 0 & 0 & 0 & 0 & 0
\end{array}\right), \quad \varphi=\left(\begin{array}{rrrrrr}
-1 & 1 & & & & \\
& -1 & 1 & & & \\
& & -1 & 1 & & \\
& & & -1 & 1 & \\
& & & & -1 & 1
\end{array}\right), \\
& \pi=\left(\begin{array}{llllll}
0 & 1 & 0 & 0 & 0 & 0 \\
0 & 0 & 1 & 0 & 0 & 0 \\
0 & 0 & 0 & 0 & 0 & 1 \\
0 & 0 & 0 & 1 & 0 & 0 \\
0 & 0 & 0 & 0 & 1 & 0 \\
1 & 0 & 0 & 0 & 0 & 0
\end{array}\right)
\end{aligned}
$$


We have

$$
K_{0}\left(\mathcal{O}_{A_{\mathscr{K}}(f \mu)}\right) \cong \mathbb{Z}_{2}, \quad K_{1}\left(\mathcal{O}_{A_{\mathscr{K}}(f \mu)}\right) \cong\{0\}
$$

REMARK 3. In the statement of Theorem 1 the case $a=0$ may occur. This happens when we have a star product factorizable kneading sequence [4]. In this case the correspondent Markov transition matrix is reducible.

REMARK 4. In [6], Katayama et al. have constructed a class of $C^{*}$-algebras from the $\beta$-expansions of real numbers. In fact, considering a semiconjugacy from the real quadratic map to the tent map [14], we can also obtain Theorem 1 using [6] and the $\lambda$-expansions of real numbers introduced in [4].

REMARK 5. In [13] (see also [12]) and [11], the BF-groups are explicitly calculated with respect to another kind of maps on the interval.

\section{REFERENCES}

[1] R. Bowen and J. Franks, Homology for zero-dimensional nonwandering sets, Ann. of Math. (2) 106 (1977), no. 1, 73-92.

[2] J. Cuntz, A class of $C^{*}$-algebras and topological Markov chains. II. Reducible chains and the Ext-functor for $C^{*}$-algebras, Invent. Math. 63 (1981), no. 1, 25-40.

[3] J. Cuntz and W. Krieger, A class of $C^{*}$-algebras and topological Markov chains, Invent. Math. 56 (1980), no. 3, 251-268.

[4] B. Derrida, A. Gervois, and Y. Pomeau, Iteration of endomorphisms on the real axis and representation of numbers, Ann. Inst. H. Poincaré Sect. A (N.S.) 29 (1978), no. 3, 305-356.

[5] J. Guckenheimer, Sensitive dependence to initial conditions for one-dimensional maps, Comm. Math. Phys. 70 (1979), no. 2, 133-160.

[6] Y. Katayama, K. Matsumoto, and Y. Watatani, Simple $C^{*}$-algebras arising from $\beta$-expansion of real numbers, Ergodic Theory Dynam. Systems 18 (1998), no. 4, 937-962.

[7] J. P. Lampreia and J. Sousa Ramos, Trimodal maps, Internat. J. Bifur. Chaos Appl. Sci. Engrg. 3 (1993), no. 6, 1607-1617.

[8] _ Symbolic dynamics of bimodal maps, Portugal. Math. 54 (1997), no. 1, $1-18$.

[9] S. Lang, Algebra, Addison-Wesley, Massachusetts, 1965.

[10] D. Lind and B. Marcus, An Introduction to Symbolic Dynamics and Coding, Cambridge University Press, Cambridge, 1995.

[11] N. Martins, R. Severino, and J. Sousa Ramos, Bowen-Franks groups for bimodal matrices, J. Differ. Equations Appl. 9 (2003), no. 3-4, 423-433.

[12] N. Martins and J. Sousa Ramos, Cuntz-Krieger algebras arising from linear mod one transformations, Differential Equations and Dynamical Systems (Lisbon, 2000), Fields Inst. Commun., vol. 31, American Mathematical Society, Rhode Island, 2002, pp. 265-273.

[13] _ Bowen-Franks groups associated with linear mod one transformations, to appear in Internat. J. Bifur. Chaos Appl. Sci. Engrg., 2003. 
[14] J. Milnor and W. Thurston, On iterated maps of the interval, Dynamical Systems (College Park, Md, 1986-87), Lecture Notes in Math., vol. 1342, Springer, Berlin, 1988, pp. 465-563.

[15] P. Štefan, A theorem of Šarkovskii on the existence of periodic orbits of continuous endomorphisms of the real line, Comm. Math. Phys. 54 (1977), no. 3, 237248.

Nuno Martins: Departamento de Matemática, Instituto Superior Técnico, 1049-001 Lisboa, Portugal

E-mail address: nmartins@math.ist.ut1.pt

Ricardo Severino: Departamento de Matemática, Universidade do Minho, 4710-057 Braga, Portugal

E-mail address: ricardo@math.uminho.pt

J. Sousa Ramos: Departamento de Matemática, Instituto Superior Técnico, 1049-001 Lisboa, Portugal

E-mail address: sramos@math.ist.ut1.pt 


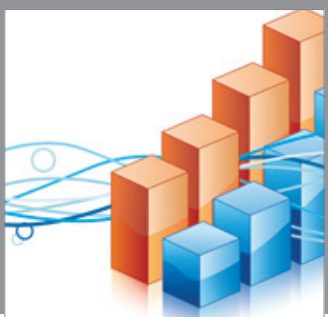

Advances in

Operations Research

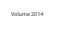

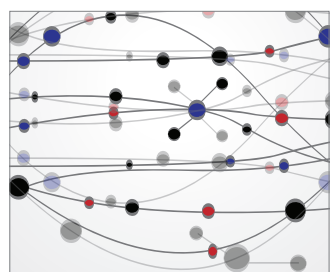

\section{The Scientific} World Journal
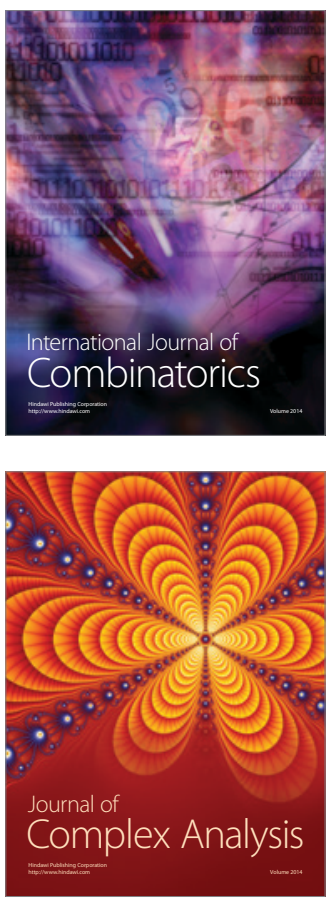

International Journal of

Mathematics and

Mathematical

Sciences
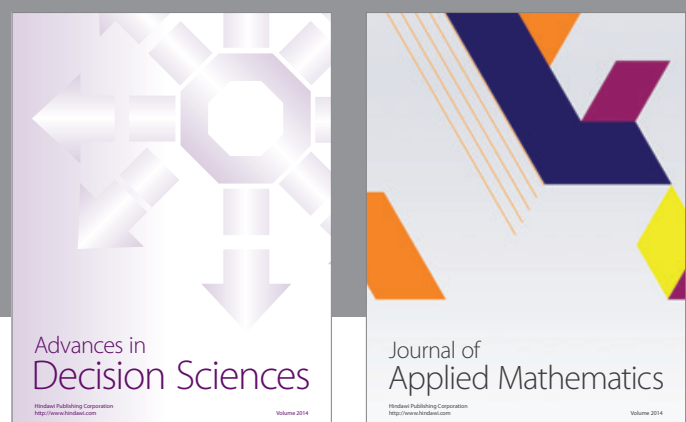

Journal of

Applied Mathematics
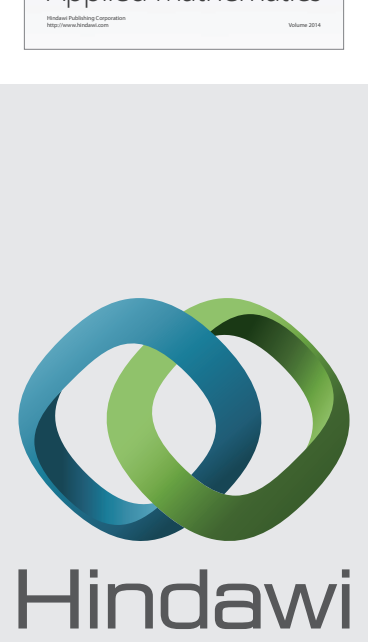

Submit your manuscripts at http://www.hindawi.com
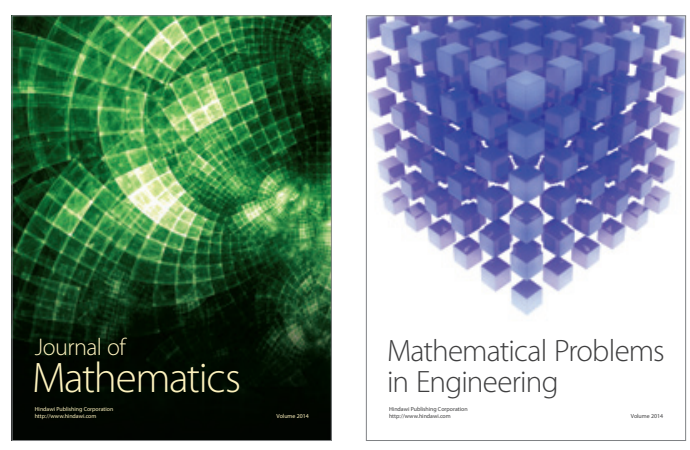

Mathematical Problems in Engineering
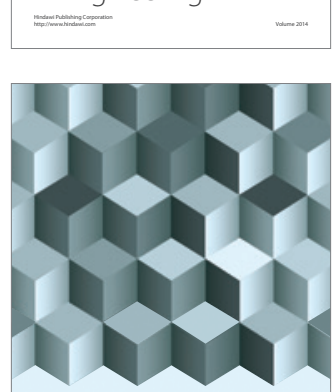

Journal of

Function Spaces
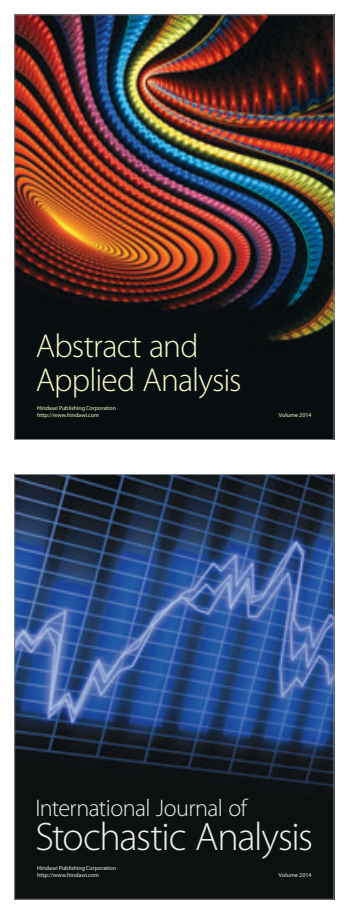

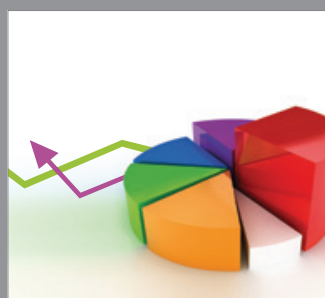

ournal of

Probability and Statistics

Promensencen
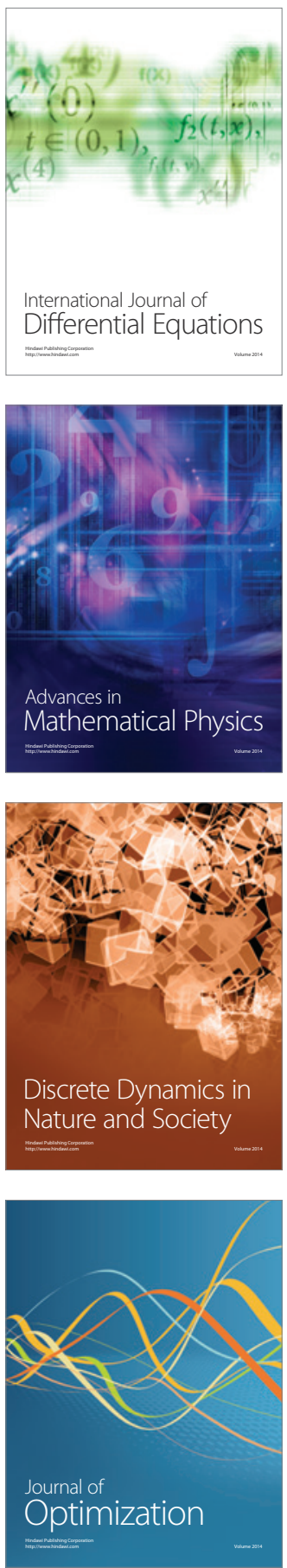